should try to be more realistic and sceptical about his administration; the new team will make mistakes, too, and should hear about them when it does. And researchers should remember that political winds can change. The Republicans will at some point be in the majority again, and lines of communication need to be kept open.

Perhaps most importantly, scientists must not fall into the trap of believing that their research necessarily supports a single course of action - partisan or otherwise. Scientists can tell policy-makers what the consequences might be if, say, atmospheric concentrations of carbon dioxide were to stabilize at 400 or 600 or 1,000 parts per million. But they cannot tell policy-makers what level the world should work towards, because that depends on what consequences people are prepared to accept - especially when weighed against economic or energy needs. Such a decision is a political matter. So when scientists are acting as advisers, they must be willing and able to set their personal preferences aside and examine a range of possible outcomes. And when they are speaking on their own behalf — as is their right as citizens - they must convey such an endorsement as opinion, not as a scientific finding.

As a corollary, researchers must also continue to speak out against politicians who pick and choose data to support their preconceived conclusions, as the Bush administration was accused of doing when it systematically played down evidence for climate change, or for the ineffectiveness of abstinence-based sex education. Wise policy, like good science, is possible only when decision-makers look at all the facts - and understand all the uncertainties in those facts.

For thousands of researchers in the United States and abroad, Obama's words promise a new era of openness and an elevation of science in political life. Science may be restored to its rightful place, but it will be up to scientists to ensure that it stays there.

\section{A responsibility index}

\section{How to evaluate a nation's scientific integrity.}

I there was one word that resonated above all at this week's Global Competitiveness Forum in Riyadh, Saudi Arabia, it was 'trust'. Given the context of the meeting - a disastrous meltdown of the world financial system - the assembled chief executives of international companies and institutions might have found this something of a sick joke. But Saudi Arabia has set itself the goal of strong international competitiveness, and in the past year has climbed the rankings of competitiveness as measured by the World Economic Forum from 35 th to 27 th position. It also wants to promote a national climate and international perception of good corporate behaviour - a goal in which trust is essential.

Like many emerging countries, Saudi Arabia measures itselfby indices, and has developed its own index for 'responsible competitiveness', based on a number of metrics (see www.rci.org.sa). But fostering strong science-based innovation requires its own metrics of inputs and achievement. So here, for any country concerned about the reputation and integrity of its research base, are some metrics that might be developed into an index for responsible scientific competitiveness.

One set of metrics relates to misconduct such as fraud, fabrication and plagiarism, which can gravely damage a country's reputation and destroy that of any researcher caught in its immediate wake, let alone the perpetrators. Two responsibility metrics would therefore relate to the infrastructures in place for the prevention and to the investigation, punishment and open declaration of misconduct. The latter metric, in turn, would look at the investigatory mechanisms in place at both the national funding-agency level, and the local level of publicly funded universities and government labs.

Less headline-grabbing misbehaviour is also important. As discussions about scientifically developed countries in this publication have shown, human nature and pressures to deliver results lead to worrying levels of discreet sleaze - the selection or cleaning up of data, the addition or removal of names from author lists and the like. This can only be treated in culture, by the education of young researchers in good practice, by the reward of good mentoring, by the scrutiny and preservation of lab notebooks, and the insistent emphasis of guidelines. Such institutional encouragement of good practice is easy to document as a metric, albeit in short supply in most countries.

A second set of metrics would measure the transparency and objectivity of a nation's systems of evaluation, funding, staff appointments and promotion. A system that discriminates against researchers on the grounds of gender, ethnicity, age or cronyism is short-sighted and will gravely undermine the scientific potential of any nation.

A third set would evaluate a nation's framework for science policy, and the extent to which it allows talented scientists to follow their noses in the pursuit of what makes the world tick while also giving societal values and economic needs their due priority.

And a final set would measure the elusive concept of 'openness' - a key corollary of trust. Openness implies a receptivity to the ideas and practices of researchers in other countries, especially valuable given the evidence that international collaborations are more powerful generators of strong science than those that are intra-national. But openness is also expressed as a willingness to have ideas and conclusions publicly criticized - a culture essential to science itself, but also to the successful pursuit of a robust strategic policy and hence to international confidence.

Taken together, these qualitative metrics would amount to an index of responsible science for any country, whatever its stage of scientific development. They could be measured by the documentation of structures and practices and by independent surveys of scientists. A study of the state of openness and development in Arab countries (N. Fergany Nature 444, 33-34; 2006) showed that Saudi Arabia in 2005 was starting from a low base. Since then, support for education, uptake by women of tertiary education and support for science have experienced significant growth. We leave it to the Saudis and to researchers and policy-makers in other ambitious technological powers to reflect on just how well their countries measure up across these metrics of responsible scientific competitiveness. 